\section{Ene Sigports}

\section{Percutaneous transhepatic hepatico- gastrostomy for portal biliopathy: anovel approach}

\section{Introduction}

Portal biliopathy $(\mathrm{PB})$ denotes an abnormality of the biliary tree in a patient with portal hypertension. It is predominantly seen in those with extrahepatic portal vein obstruction $(\text { EHPVO })^{1}$ a common cause of portal hypertension in India. ${ }^{2}$ Most patients with portal biliopathy are asymptomatic but some develop obstructive jaundice with or without cholangitis. The treatment options for symptomatic portal biliopathy are endoscopic retrograde cholangiography (ERC) and stenting, portosystemic shunts or bilio-enteric drainage. However biliary decompression in these patients is often difficult and hazardous. They are at risk of bleeding ${ }^{1}$ during endoscopic sphincterotomy; ten percent of patients with EHPVO have veins that are not amenable for shunting; ${ }^{3}$ operative bilio-enteric drainage is dangerous due to the presence of extensive collaterals and we have noticed that the biliopathy sometimes persists, perhaps due to ischaemic bile duct strictures, in spite of adequate portal decompression via a patent portosystemic anastomosis. Thus there is a small group of patients with portal biliopathy in whom biliary decompression seems not to be possible. In one such patient we used a novel approach to decompress the biliary obstruction resulting from portal hypertension which we describe here.

\section{Case report}

A twenty eight year old male patient presented with painless jaundice 15 years ago at another hospital. Investigations revealed a distal bile duct stricture. Initially ERC was tried twice, but failed. An attempt was made to perform a choledochojejunostomyby open surgery but was abandoned because of major bleeding from extensive collaterals around the portahepatis. A cholecystojejunostomy was fashioned instead. After a transient improvement for a month, the jaundice recurred and continued in intermittent episodes. He presented to us two years ago with obstructive jaundice with serum bilirubin $17.2 \mathrm{mg} / \mathrm{dl}$ and serum alkaline phosphatase $423 \mathrm{IU} / \mathrm{L}$.
Investigations carried out included CT angiography which revealed extensive thrombosis involving the portal, superior mesenteric and splenic veins with a portal cavernoma (Figure 1). Magnetic resonancecholangiopancreatography (MRCP) showed narrowing of the mid-bile duct with dilatation of the proximal radicals (Figure 2). ERC was attempted but abandoned due to bleeding from the papilla. Due to the extensive splenoportal venous thrombosis, a portosytemic shunt was not possible, so we attempted a direct hepaticojejunostomy. At exploration, we found dense adhesions and extensive collaterals in the abdomen and around the porta. After dismantling the previous cholecystojejunostomy which was

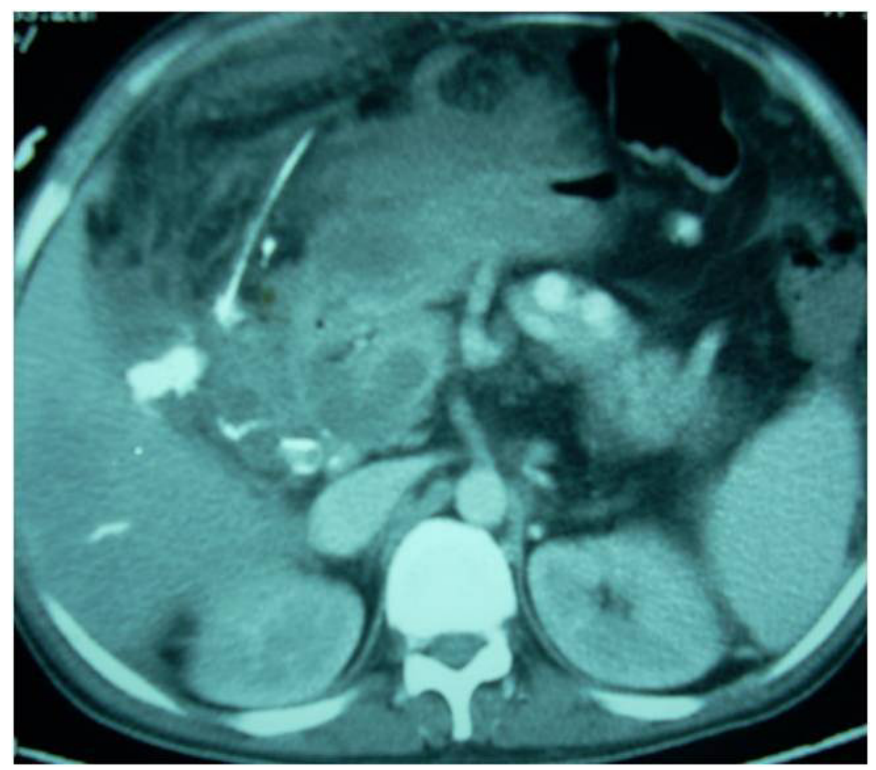

Figure 1: Computed tomography showing splenoportal axis thrombosis with collaterals

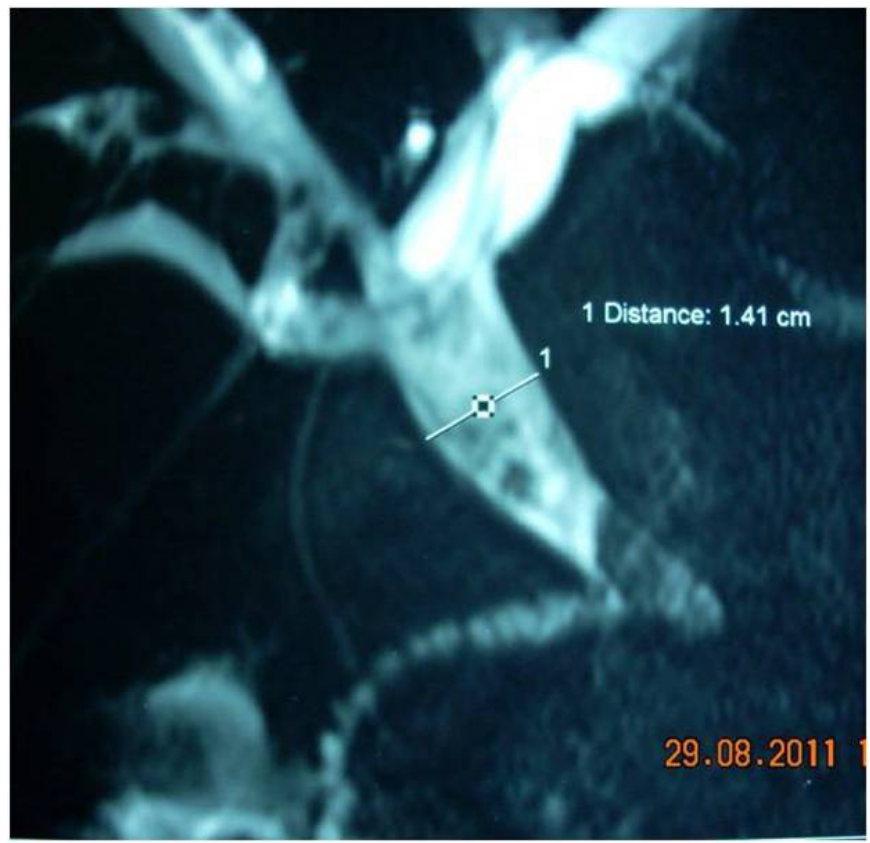

Figure 2: Magnetic resonant cholangiography showing mid bile duct narrowing 
no longer patent we encountered massive bleeding and hemodynamic instability during adhesiolysis and porta dissection. A small segment of bile duct was seen just below the confluence. In view of the instability, a T-tube was placed in the bile duct and abdominal packing was done to control bleeding. These packs were removed after 48 hours. Postoperatively the patient required prolonged ventilation and an ICU stay of 15 days. He was discharged 20 daysafter operation with the T-tube in-situ. However, after a month he was re-admitted with fever and investigations revealed a blocked T-tube with an intra-abdominal collection. The T-tube was flushed and an ultrasound guided drain was placed in the abdomen which drained bile for several days. Again after three months, the T-tube stopped draining and the patient developed obstructive jaundice. T-tube cholangiography showed complete cut off at the mid-bile duct and ultrasound showed dilated intrahepatic biliary radicals. Right anterior segment percutaneous transhepatic biliary drainage (PTBD) was performed and an external catheter was placed. We then explored the patient again to try and perform a bilio-enteric anastomosis and provide definitive treatment. However the upper abdomen was completely frozen and there were extensive collaterals. Massive bleeding was encountered and surgery was abandoned. Later internalization of the external PTBD catheter into the duodenum was attempted, but due to bleeding from the bile duct, the procedure was abandoned.We were thus faced with a difficult situation in a young patient with portal biliopathy in whom biliary decompressive procedures had been unsuccessful and portosystemic shunts were not an option. We then decided to try and drain the biliary system into the stomach via the percutaneous route.

\section{Procedure}

A guide wire was passed through the previously placed right anterior PTBD catheter, across the confluence up to the segment II duct, which was the closest to the stomach wall. The stomach was distended with air and gastroparesis was achieved using intravenous $20 \mathrm{mg}$ of hyoscinebutylbromide. A needle was passed over a guide wire to the segment II duct and advanced into the stomach by piercing its wall. This was confirmed by injecting contrast (Figure 3). Through the needle sheath a super stiff wire was placed in the stomach and the track between the segment II duct of the liver and stomach lumen dilated with an $8 \mathrm{~mm}$ angioplasty balloon catheter. A 10 $\mathrm{cm}$ long, $10 \mathrm{~F}$, self expanding covered metal stent was placed from the segment II bile duct into the stomach lumen, $4 \mathrm{~cm}$ of which was kept lying within the gastric lumen to prevent displacement during peristalsis (Figure 4). The procedure was uneventful and took 45 minutes. After 48 hours a check cholangiogram from the right PTBD catheter showed free drainage of contrast into the stomach without any extra-luminal leak (Figure 5), so the external right PTBD catheter and T-tube were removed.

\section{Results}

The post procedure recovery was uneventful with oral diet being started 6 hours later. Serial serum bilirubin and alkaline

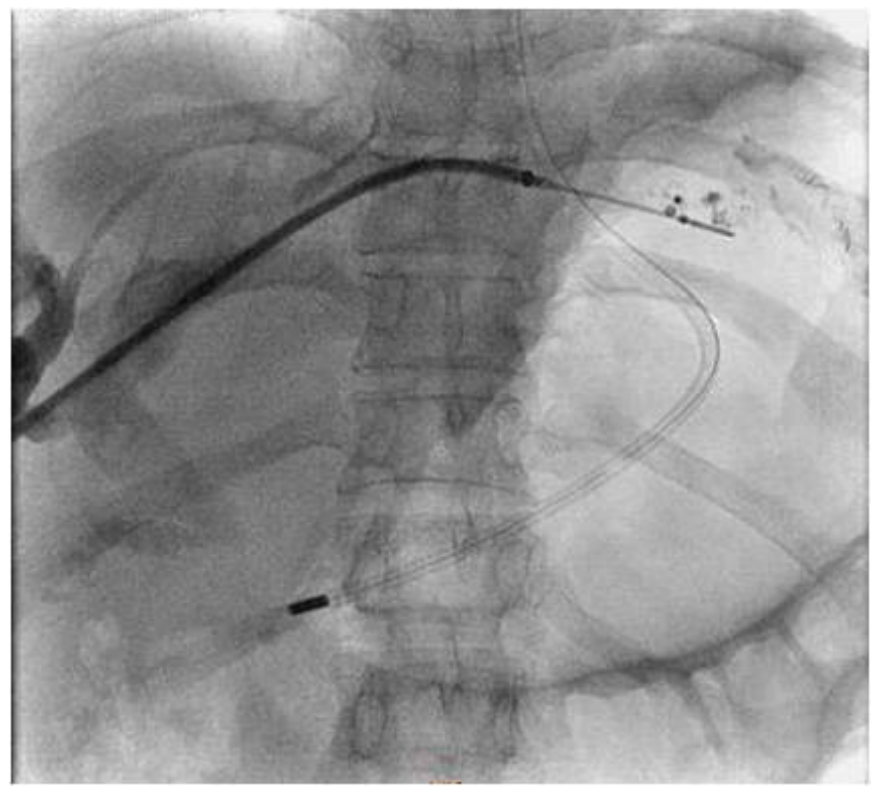

Figure 3: Percutaneous transhepatichepaticogastrostomy procedure. Needle with guide wire pierced from segment II duct to gastric lumen. Ryle's tube in stomach.

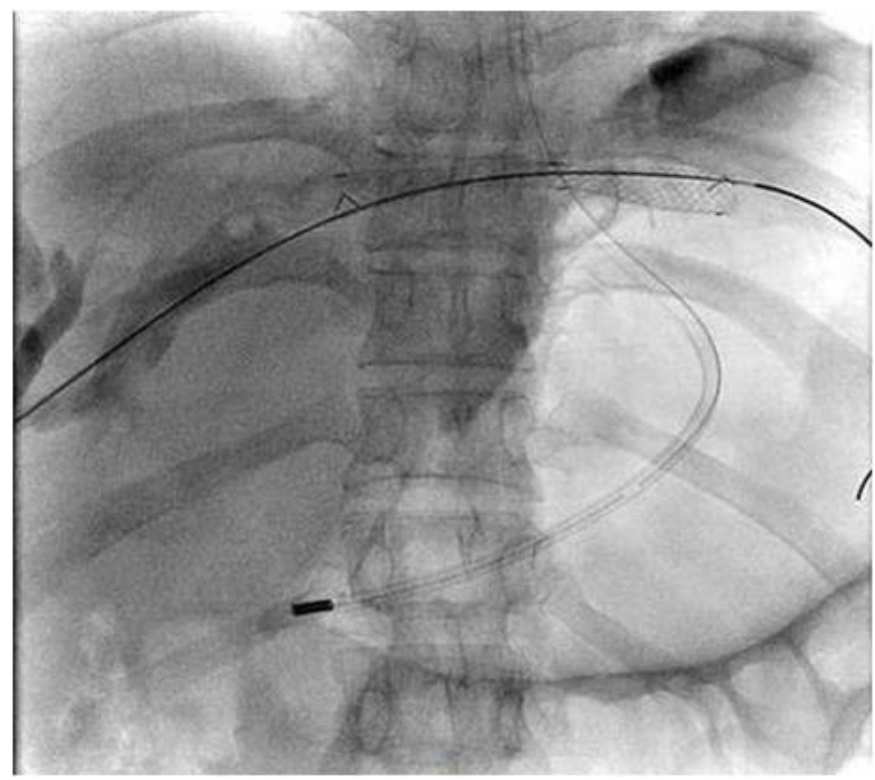

Figure 4: Percutaneous transhepatichepaticogastrostomy procedure. Note self expanding metallic stent over guide wire. 


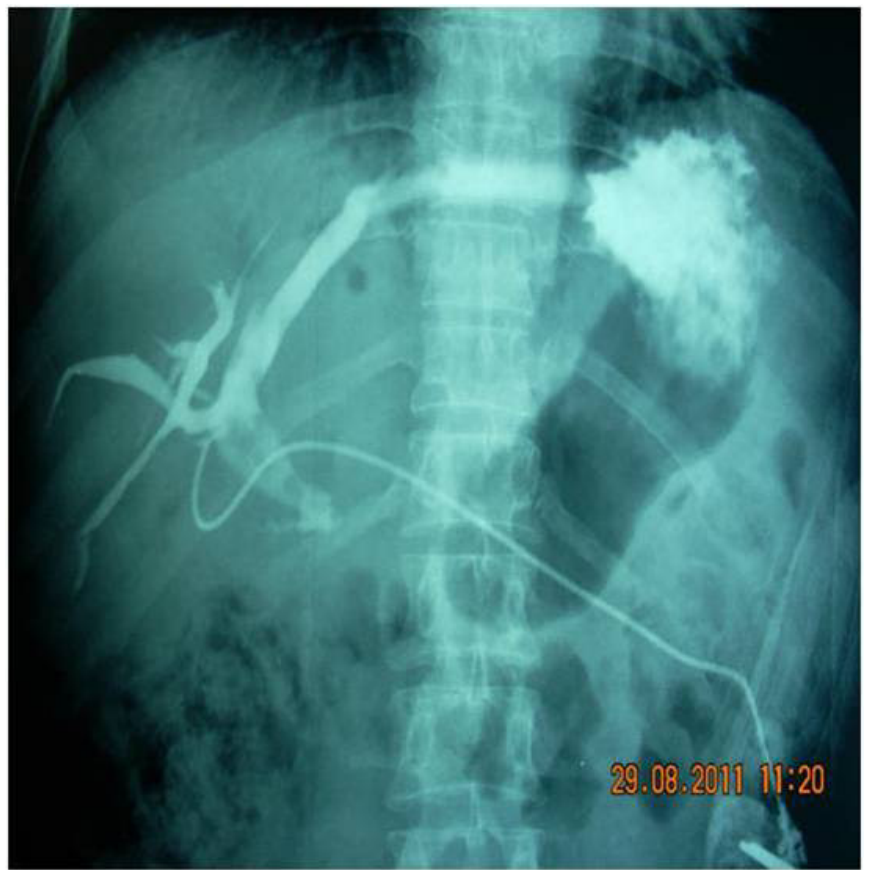

Figure 5: Check cholangiogram showing free flow of contrast into stomach without leak

phosphatase showed a decreasing trend.He was discharged on day 6 after the procedure and now at a 6 month follow-up, the patient is doing well with no episodes of cholangitis, blockage or migration of the stent. His last serum bilirubin level was $2.1 \mathrm{mg} / \mathrm{dl}$.

\section{Discussion}

The exact mechanism of portal biliopathy is not known but is probably due to obstruction of the bile duct by a portal cavernoma pressing on the bile duct from outside, pericholedochol varices within the wall or a fibrous stricture due to ischemia. ${ }^{1}$ On routine ERC,cholangiographic abnormalities of the biliary tree have been reported in $80-100 \%$ cases of patients with EHPVO, ${ }^{4-7}$ but symptoms of portal biliopathy occur only in $5-14 \%$ of the cases. ${ }^{4,6,8,9}$

Patients with obstructive jaundice and cholangitis from portal biliopathy should be initially treated with ERC and stenting. However this may be hazardous as these patients may bleed profusely from collaterals around the papilla. ${ }^{1}$ Patients without cholangitis and with veins amenable to shunting, should be treated with a portosystemic shunt. ${ }^{2}$ The majority of patients improve after shunt surgery alone but about one third are not relieved of biliary obstruction following surgery, probably because of ischemic stricture and scarring ${ }^{6}$ and require bilio-enteric drainage. ${ }^{10,11}$ These patients should be treated with a surgical bilio-enteric anastomosis as their long term outcome is not satisfactory with endoscopic treatment. ${ }^{10}$ Surgical bilio-enteric drainage is easier after portosystemic shunt due to reduction in portal pressure in the collaterals.

Ten percent of patients with EHPVO have extensive splenoportal venous thrombosis, ${ }^{3}$ where neither endoscopic treatment nor a surgical bilio-enteric bypass can be offered, as was the situation in our patient. Endoscopic treatment was also not an option as he bled profusely from the ampulla during a previous ERC. Two attempts at surgical decompression failed due to life-threatening hemorrhage from extensive collaterals. Internalization of the external PTBD catheter across the bile duct stricture into the duodenum also failed due to bleeding. We therefore decided to attempt a percutaneous transhepatichepaticogastrostomy. Similar techniques have been described for unresectable biliary malignancies, but there has been no report of its use in portal biliopathy.

Gilles et a ${ }^{12}$ reported 35 cases of hepaticogastrostomy under fluoroscopic, endoscopic and laparoscopic guidance with a mean patency of 234 days and a jaundice free survival rate of $100 \%, 96 \%, 93 \%$ and $80 \%$ at $1,3,6$, and 12 months, respectively. The authors used glue to seal the anastomosis and observed no leak in their series. Periprocedure cholangitis occurred in $11 \%$ and late cholangitis occurred in $20 \%$, mainly due to exclusion of the right bile duct. A few patients developed bile gastritis, which was controlled with omeprazole. Tipaldiet $\mathrm{al}^{13}$ reported a modified technique of performing percutaneous hepaticogastrostomy without laparoscopy in patients with malignant biliary obstruction. The authors used the Cope anchor system under CT guidance which develops bonding between the left lobe of the liver and the stomach after which they performed a hepaticogastrostomy. Endoscopic ultrasound(EUS) guided hepaticogastrostomy has been described in several studies. Most of the reports have been for unresectable malignancy. Park et al ${ }^{14}$ performed EUS guided hepaticogastrostomy using fully covered self expandable metal stents in 14 patients with malignant biliary obstruction with a $100 \%$ technical success rate. One patient required reintervention for stent migration and there was no incidence of stent occlusion with a median follow up of 6 months.

We successfully performed percutaneous transhepatichepaticogastrostomy in a patient with portal biliopathy in whom no other treatment options were available. We used a self expanding metal stent to reduce the risk of occlusion, dilated the track with an $8 \mathrm{~mm}$ balloon catheter and placed a $10 \mathrm{~F}$ stent, $4 \mathrm{~cm}$ of which was kept inside the gastric 
lumen to prevent stent migration during peristalsis.

There is a concern in these patients regarding bile gastritis and meal induced cholangitis. In the absence of pancreatic secretion bile should not damage the gastric mucosa and the symptoms usually settle with proton pump inhibitors. Ramesh et $\mathrm{al}^{15}$ reported surgical hepaticogastrostomy without bile gastritis or gastric acid induced cholangitis. The other potential complications are bile leak, bleeding, hemobilia and stent migration which did not occur in our case. To the best of our knowledge, this is the first report of percutaneous hepaticogastrostomy in a patient with portal biliopathy in whom surgical attempts at biliary decompression had failed and portosystemic shunting was not possible.

\section{JITENDRA H MISTRY ${ }^{1}$ VIBHA VARMA ${ }^{1}$ NAIMISH MEHTA ${ }^{1}$ VINAY KUMARAN ${ }^{1}$ SAMIRAN NUNDY ${ }^{1}$} ARUN GUPTA ${ }^{2}$

Correspondence: Dr. Jitendra H Mistry Departments of Surgical Gastroenterology and Liver Transplantation ${ }^{1}$ and Intervention Radiology ${ }^{2}$ Sir Ganga Ram Hospital, New Delhi - 110060, India Email:jitlap@gmail.com

\section{References}

1. Chandra R, Kapoor D, Tharakan A, Chaudhary A, Sarin SK. Portal biliopathy. J Gastroenterol Hepatol. 2001;16:1086-92.

2. Dhiman RK, Behera A, Chawla YK, Dilawari JB, Suri S. Portal hypertensive biliopathy. Gut. 2007;56:1001-8.

3. Mitra SK,Kumar V, Dutta DV, Rao PN, Sandhu K, Singh GK, Sodhi JS, Pathak IC. Extrahepatic portal vein hypertension: a review of 70 cases. J Pediatr Surg. 1978;13:51-7.

4. Dilawari JB, Chawla YK. Pseudosclerosing cholangitis in extrahepatic portal venous obstruction. Gut. 1992;33:272-6.

5. Sarin SK, Bhatia V, Makwane U. Portal biliopathy in extrahepatic portal vein obstruction. Indian J Gastroenterol. 1992;11:82.

6. Khuroo MS, Yatoo GN, Zargar SA, Javid G, Dar MY, Khan BA, et al. Biliary abnormalities associated with extrahepatic portal venous obstruction. Hepatology. 1993;17:807-13.

7. Chandra R, Tharakan A, Kapoor D, Sarin SK. Comparative study of portal biliopathy in patients with portal hypertension due to different etiologies. Indian J Gastroenterol. 1997;15:A59.

8. Condat B, Vilgrain V, Asselah T, O'Toole D, Rufat P, Zappa M, et al. Portal cavernoma-associated cholangiopathy: a clinical and MR cholangiography coupled with MR portography imaging study. Hepatology. 2003;37:1302-8.

9. Malkan GH, Bhatia SJ, Bashir K, Khemani R, Abraham P, Gandhi MS,et al,Cholangiopathy associated with portal hypertension: diagnostic evaluation and clinical implications. Gastrointest Endosc. 1999;49:344-8.
10. Agarwal AK, Sharma D, Singh S, Agarwal S, Girish SP. Portal biliopathy: a study of 39 surgically treated patients. HPB(Oxford). 2011;13:33-9.

11. Chaudhary A, Dhar P, Sarin SK, Sachdev A, Agarwal AK, Vij JC, et al. Bile duct obstruction due to portal biliopathy in extrahepatic portal hypertension: surgical management. Br J Surg. 1998;85:326-9.

12. Soulez G, Gagner M, Thérasse E, Deslandres E, Pomp A, Leduc $\mathrm{R}$, et al. Malignant biliary obstruction: preliminary results of palliative treatment with hepaticogastrostomy under fluoroscopic, endoscopic, and laparoscopic guidance. Radiology. 1994;192:241-6.

13. Tipaldi L. A simplified percutaneous hepatogastric drainage technique for malignant biliary obstruction. Cardiovasc Intervent Radiol. 1995;18:333-6.

14. Park do H, Song TJ, Eum J, Moon SH, Lee SS, Seo DW, et al. EUS-guided hepaticogastrostomy with a fully covered metal stent as the biliary diversion technique for an occluded biliary metal stent after a failed ERCP (with videos). Gastrointest Endosc. 2010;71:413-9.

15. Ramesh H. Intrahepatic cholangiogastrostomy for malignant biliary obstruction at the hilum. Br J Surg. 1992;79:1349-50.

\section{Segmental ileal dilatation: an unsuspected cause of neonatal intestinal obstruction}

\section{Introduction}

Segmental dilatation of small intestine although described in literature, is among the rare causes of intestinal obstruction in neonates. The etiology of this condition remains unknown. ${ }^{1}$ Clinical presentation in these patients is like any other intestinal obstruction but investigations often reveal a confusing picture, thereby arousing clinical suspicion of this condition. Once the diagnosis is confirmed resection of the affected segment is the treatment of choice. We present here a case of neonatal intestinal obstruction due to segmental ileal dilatation and discuss the pertinent clinical aspects of this condition with a relevant review of literature.

\section{Case report}

We present here a female child born to a primigravida mother at 37 weeks of gestation with birth weight of $2.2 \mathrm{~kg}$. The baby 